\title{
Perioperative complications with multilevel anterior and posterior cervical decompression and fusion
}

\author{
Joshua T. Wewel, MD,, Bledi C. Brahimaj, MD, ${ }^{1}$ Manish K. Kasliwal, MD, MCh, ${ }^{2}$ and \\ Vincent C. Traynelis, MD' \\ 'Department of Neurosurgery, Rush University Medical Center, Chicago, Illinois; and 2Department of Neurosurgery, University \\ Hospital Cleveland Medical Center, Case Western Reserve University School of Medicine, Cleveland, Ohio
}

\begin{abstract}
OBJECTIVE Cervical spondylotic myelopathy (CSM) is a progressive degenerative pathology that frequently affects older individuals and causes spinal cord compression with symptoms of neck pain, radiculopathy, and weakness. Anterior decompression and fusion is the primary intervention to prevent neurological deterioration; however, in severe cases, circumferential decompression and fusion is necessary. Published data regarding perioperative morbidity associated with these complex operations are scarce. In this study, the authors sought to add to this important body of literature by documenting a large single-surgeon experience of single-session circumferential cervical decompression and fusion.
\end{abstract}

METHODS A retrospective analysis was performed to identify intended single-stage anterior-posterior or posterioranterior-posterior cervical spine decompression and fusion surgeries performed by the primary surgeon (V.C.T.) at Rush University Medical Center between 2009 and 2016. Cases in which true anterior-posterior cervical decompression and fusion was not performed (i.e., those involving anterior-only, posterior-only, or delayed circumferential fusion) were excluded from analysis. Data including standard patient demographic information, comorbidities, previous surgeries, and intraoperative course, along with postoperative outcomes and complications, were collected and analyzed. Perioperative morbidity was recorded during the 90 days following surgery.

RESULTS Seventy-two patients ( 29 male and 43 female, mean age 57.6 years) were included in the study. Fourteen patients (19.4\%) were active smokers, and $56.9 \%$ had hypertension, the most common comorbidity. The most common clinical presentation was neck pain in 57 patients (79.2\%). Twenty-three patients (31.9\%) had myelopathy, and 32 patients (44.4\%) had undergone prior cervical spine surgery. Average blood loss was $613 \mathrm{ml}$. Injury to the vertebral artery was encountered in 1 patient (1.4\%). Recurrent laryngeal nerve palsy was observed in 2 patients (2.8\%). Two patients $(2.8 \%)$ had transient unilateral hand grip weakness. There were no permanent neurological deficits. Dysphagia was encountered in 45 patients (62.5\%) postoperatively, with 23 (32\%) requiring nasogastric parenteral nutrition and $9(12.5 \%)$ patients ultimately undergoing percutaneous endoscopic gastrostomy (PEG) placement. Nine of the 72 patients required a tracheostomy. The incidence of pneumonia was $6.9 \%$ (5 patients) overall, and 2 of these patients were in the tracheostomy group. Superficial wound infections occurred in 4 patients (5.6\%). Perioperative death occurred in 1 patient. Reoperation was necessary in 10 patients (13.9\%). Major perioperative complications (permanent neurological deficit, vascular injury, tracheostomy, PEG tube, stroke, or death) occurred in $30.6 \%$ of patients. The risk of minor perioperative complications (temporary deficit, dysphagia, deep vein thrombosis, pulmonary embolism, urinary tract infection, pneumonia, or wound infection) was $80.6 \%$.

CONCLUSIONS Single-session anterior-posterior cervical decompression and fusion is an inherently morbid operation required in select patients with cervical spondylotic myelopathy. In this large single-surgeon series, there was a major perioperative complication risk of $30.6 \%$ and minor perioperative complication risk of $80.6 \%$. This overall elevated risk for postoperative complications must be carefully considered and discussed with the patient preoperatively. In some situations, shared decision making may lead to the conclusion that a procedure of lesser magnitude may be more appropriate.

https://thejns.org/doi/abs/10.3171/2019.6.SPINE198

KEYWORDS cervical; complication; decompression; fusion; dysphagia; perioperative

ABBREVIATIONS CLT = cuff-leak test; COPD = chronic obstructive pulmonary disease; $C S M=$ cervical spondylotic myelopathy; DVT = deep vein thrombosis; PE = pulmonary embolism; PEG = percutaneous endoscopic gastrostomy; RLN = recurrent laryngeal nerve; UTI = urinary tract infection.

SUBMITTED January 3, 2019. ACCEPTED June 28, 2019.

INCLUDE WHEN CITING Published online September 20, 2019; DOI: 10.3171/2019.6.SPINE198. 


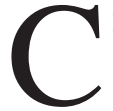
ERVICAL spine degenerative pathologies, which include cervical spondylotic myelopathy (CSM), remain among the most common spinal conditions treated by spine surgeons worldwide. CSM often affects older individuals, and the spinal cord or nerve root compression may present with a number of signs and symptoms, including neck pain, radiculopathy, sensory changes, balance issues, weakness, and severe myelopathy. ${ }^{11,20}$ Less commonly, cervical deformity may be encountered. Kyphosis is the primary degenerative deformity but there is often anterior-posterior translation at one or more segments as well. ${ }^{16}$ Predisposing factors for kyphosis include trauma, infection, congenital anomalies, and poorly conceived or executed prior surgical procedures. Patients with CSM and kyphosis will deteriorate neurologically without adequate neural decompression, deformity correction, and long-term stabilization. ${ }^{16,20,21}$

Clinical data support anterior decompression and fusion as the prime treatment for symptomatic cervical kyphosis and stenosis., 2,5,22 Although sufficient, direct decompression obtained from an anterior approach alone comes at the risk of inadequate stabilization and increased potential for pseudarthrosis when 3 or more levels are involved and kyphosis correction must be maintained., ${ }^{1,5,23}$ Additionally, in select patients, both anterior and posterior decompression are required to adequately free the spinal cord. Thus, there is the need for staged or single-surgery anterior and posterior decompression and fusion in a subgroup of patients with CSM. However, few data have been published regarding perioperative morbidity associated with these complex operations.

To date, the largest patient cohorts in which perioperative complications of anterior-posterior cervical fusions have been investigated are those reported by Smith et al., ${ }^{21}$ Chen et al., ${ }^{3}$ and Siemionow et al., ${ }^{18}$ with 29, 30, and 35 patients, respectively. We present a single-surgeon experience to add to this important body of literature.

\section{Methods \\ Patient Selection}

After Institutional Review Board approval was obtained, a retrospective review was performed to identify all singlestage anterior-posterior or posterior-anterior-posterior cervical spine decompression and fusion surgeries performed by the primary surgeon (V.C.T.) at Rush University Medical Center. All cases performed by the primary surgeon from 2009 to 2016 were screened and anterior-posterior cervical decompression and fusion cases were identified. The primary diagnoses for all patients included myelopathy and/or radiculopathy secondary to degenerative cervical spondylosis with or without kyphotic deformity. Cases that were not true anterior-posterior cervical decompression and fusion (i.e., only anterior, only posterior, or delayed circumferential fusion) were excluded from analysis. Perioperative morbidity was recorded during the 90-day perioperative period.

\section{Surgical Technique}

All anterior cervical approaches were performed from the left side utilizing a sharp dissection technique. A table-mounted retractor was used for all anterior cervical approaches (Thompson Surgical Instruments). Anterior procedures consisted of a minimum of 3-level and maximum of 6-level discectomies and instrumentation. All patients undergoing discectomy received a Cornerstone allograft (Medtronic). Anterior corpectomies spanned 1 to 3 levels. In the cases for which a corpectomy was required, either fibular allografts or stackable cages were utilized (Medtronic). Posterior fixation techniques were tailored to the patient and included occipital plating and C2 (pars, pedicle, or laminar), lateral mass, and pedicle screw fixation.

\section{Data Collection}

Data extracted from medical chart review included standard demographic information, medical comorbidities, smoking status, prior cervical spine surgery, neck pain, myelopathy, and radiculopathy. Extracted intraoperative data included estimated blood loss, durotomy, recurrent laryngeal nerve injury, and vascular injury. Perioperative complications included new neurological deficit, deep vein thrombosis (DVT), pulmonary embolism (PE), urinary tract infection (UTI), pneumonia, superficial wound infection, dysphagia, need for Dobhoff nasogastric feeding tube (Covidien), need for percutaneous endoscopic gastrostomy (PEG) tube, duration of postoperative intubation, need for tracheostomy, need for reoperation, stroke, and death. Major postoperative complications were defined as tracheostomy, PEG tube, permanent neurological deficit, recurrent laryngeal nerve (RLN) injury, vascular injury, stroke, or death. Minor complications were defined as a temporary neurological deficit, durotomy, dysphagia, temporary Dobhoff tube, DVT, PE, UTI, or superficial wound infection.

\section{Extubation Criteria}

All patients left the operating room intubated following surgery and remained so at least overnight as has always been the senior author's practice. This protocol is followed because of concern regarding the potential for significant airway edema, which may occur following a large anterior dissection after which the prone position places the anterior surgical field in a dependent position that may facilitate the development of edema. After surgery, patients were transferred to the Neurosciences Intensive Care Unit. Adequate analgesia and anesthesia were provided as necessary. Airway patency was assessed using a measured and qualitative (audible or nonaudible) cuff-leak test (CLT) as originally described by Miller and $\mathrm{Cole}^{12}$ but modified for a more modern ventilation strategy that incorporates lower tidal volume ventilation ( $8 \mathrm{ml} / \mathrm{kg}$ ideal body weight), newer ventilation modes, and physiological PEEP (positive endexpiratory pressure), as these advances were introduced a posteriori. ${ }^{14}$

Expired tidal volume was recorded over 6 respiratory cycles, the cuff was reinflated, and ventilatory parameters were returned to baseline. The evaluator also assessed the cuff leak qualitatively as to whether it was audible or not. The quantitative CLT result was calculated as the difference between the mechanical inspired volume and the average of the 3 lowest tidal volumes with the cuff deflated. ${ }^{8,12}$ Patients were eligible for extubation on the following morning. Criteria for pursuing extubation included an 
awake and cooperative state, appropriate cognitive status, satisfactory ventilatory parameters (ability to maintain adequate oxygenation with an $\mathrm{FiO}_{2}$ of 0.4 ), hemodynamic stability, presence of an audible cuff leak, and CLT result equal to or greater than $200 \mathrm{ml}$.

\section{Case Example}

A typical patient included in this series who was a candidate for circumferential cervical spine surgery is shown in Fig. 1.

\section{Results}

Seventy-two patients were included in this study, 29 men and 43 women, with a composite average age of 57.6 years (range 27-82 years). All patients underwent sameday anterior-posterior or posterior-anterior-posterior procedures. Fourteen patients (19.4\%) were active smokers (6 male [20.7\%] and 8 female [18.6\%]). Hypertension was the most common comorbidity at $56.9 \%$ (41 patients) followed by hyperlipidemia in $26.4 \%$ (19 patients), asthma in $8.3 \%$ (6 patients), and chronic obstructive pulmonary disease (COPD) in $6.9 \%$ (5 patients). The most common clinical presentation was neck pain in $79.2 \%$ (57 patients). Twentythree patients $(31.9 \%)$ had myelopathy and $12(16.7 \%)$ had radiculopathy on presentation. Thirty-two patients $(44.4 \%)$ had prior cervical spine surgery (Table 1).

All patients underwent single-stage surgery. Sixty-two (86.1\%) patients underwent anterior followed by posterior fusion. Ten (13.9\%) patients underwent posterior-anterior- posterior procedures, which were necessary to perform a posterior release to allow for adequate correction. The most common caudal level of posterior instrumentation terminated at $\mathrm{T} 1$ in $42(58.3 \%)$ patients. The posterior instrumentation terminated at T2 in $17(23.6 \%)$ patients, C7 in $5(6.9 \%)$ patients, C6 in $4(5.6 \%)$ patients, C5 in $2(2.8 \%)$ patients, and $\mathrm{C} 4$ in 1 (1.4\%) patient.

Blood loss per patient from the combined anterior and posterior portions of each procedure was $613 \pm 439 \mathrm{ml}$ (mean $\pm \mathrm{SD}$ ). The length of surgery was $7.89 \pm 1.91$ hours. Injury to the vertebral artery was encountered in 1 patient $(1.4 \%)$. Recurrent laryngeal nerve palsy was observed in 2 patients $(2.8 \%)$. A dural breach was not encountered in this cohort (Table 2).

Postoperative complications resulting in a temporary neurological deficit occurred in 2 patients $(2.8 \%)$ with transient unilateral hand grip weakness. In no case did a patient have a permanent neurological deficit. Subjective dysphagia, as reported by patients, was a common finding, occurring in $45(62.5 \%)$ patients postoperatively. A temporary Dobhoff tube was necessary to supply parenteral nutrition in $23(32.0 \%)$ patients for an average of 13.3 days. A PEG tube was necessary in 9 patients, $12.5 \%$ of the entire cohort (Table 3).

The average number of days of intubation postoperatively was 3.6 days. As standard procedure for this type of surgery, all patients are left intubated overnight and their ventilator parameters assessed the next morning, and this time frame is included in the average of 3.6 days of intuba-
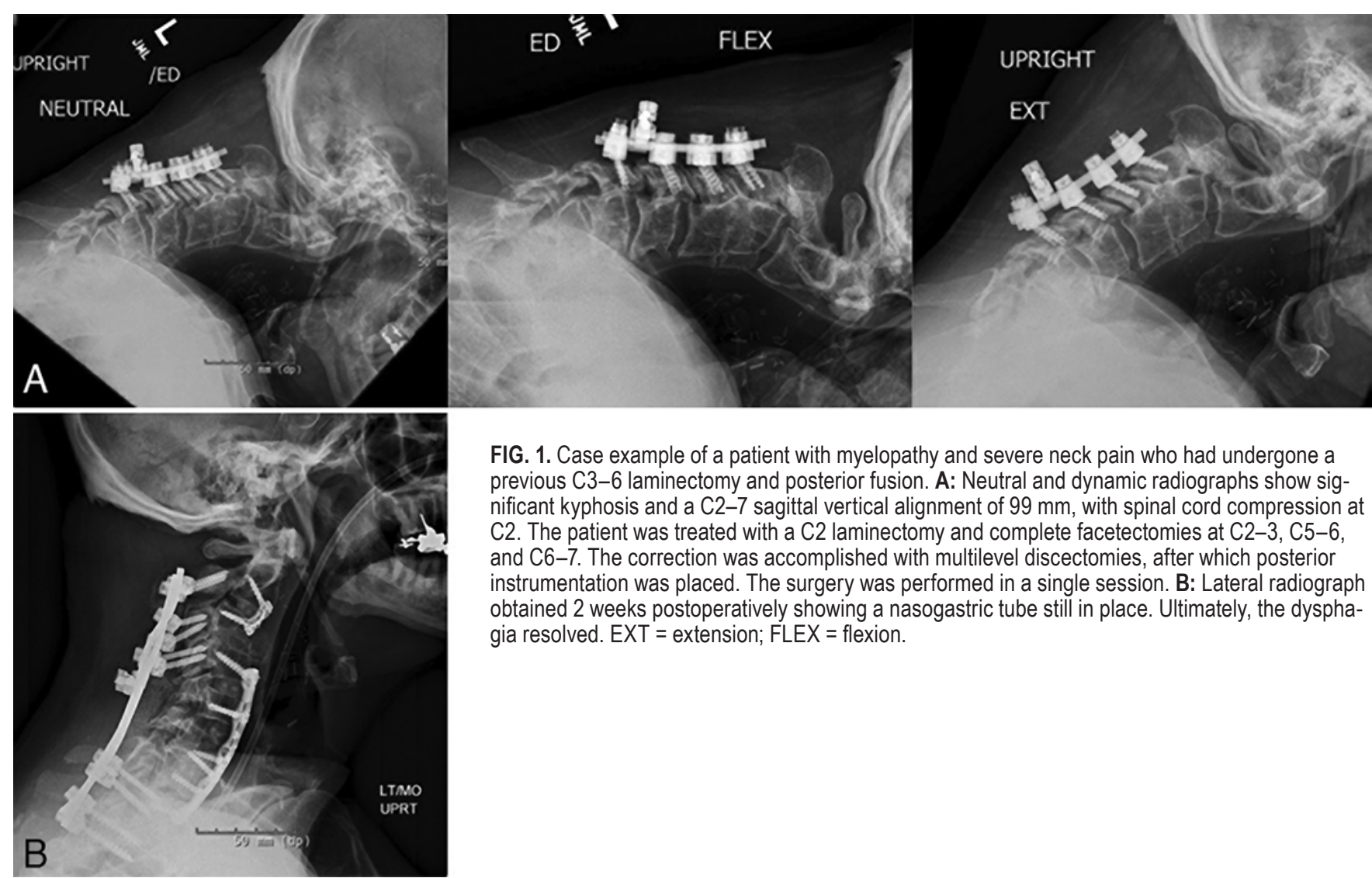

FIG. 1. Case example of a patient with myelopathy and severe neck pain who had undergone a previous C3-6 laminectomy and posterior fusion. A: Neutral and dynamic radiographs show significant kyphosis and a C2-7 sagittal vertical alignment of $99 \mathrm{~mm}$, with spinal cord compression at $\mathrm{C} 2$. The patient was treated with a C2 laminectomy and complete facetectomies at C2-3, C5-6, and $66-7$. The correction was accomplished with multilevel discectomies, after which posterior instrumentation was placed. The surgery was performed in a single session. B: Lateral radiograph obtained 2 weeks postoperatively showing a nasogastric tube still in place. Ultimately, the dysphagia resolved. $E X T=$ extension; FLEX = flexion. 
TABLE 1. Patient characteristics

\begin{tabular}{lc}
\hline & Value \\
\hline Total no. of patients & 72 \\
\hline Demographic data & \\
\hline Sex & 29 \\
\hline Male & 43 \\
\hline Female & $57.6(27-82)$ \\
\hline Age in yrs, mean (range) & $14(19.4 \%)$ \\
\hline Smoker & $57(79.2 \%)$ \\
\hline Nonsmoker & $41(56.9 \%)$ \\
\hline Hypertension & $19(26.4 \%)$ \\
\hline Hyperlipidemia & $6(8.3 \%)$ \\
\hline Asthma & $5(6.9 \%)$ \\
\hline COPD & \\
\hline Clinical presentation & $23(31.9 \%)$ \\
\hline Myelopathy & $12(16.7 \%)$ \\
\hline Radiculopathy & $57(79.2 \%)$ \\
\hline Neck pain & $32(44.4 \%)$ \\
\hline Prior surgery
\end{tabular}

Values are presented as number of patients (\%) unless otherwise indicated.

tion. Nine of the 72 patients in this study required a tracheostomy. Six of these patients were unable to be extubated per the protocol outlined in the Methods section, while 3 others were reintubated after extubation per the same guidelines. The average time of intubation to tracheostomy was 10.5 days in this cohort of patients. The incidence of pneumonia was $6.9 \%$ (5 patients) overall, and 2 of these patients were in the tracheostomy group. DVT was observed in 1 patient, and there were no patients with PE. UTI was observed in $1(1.4 \%)$ patient. Superficial wound infections occurred in $4(5.6 \%)$ patients. Stroke was not seen in any patients. Perioperative death occurred in 1 patient 6 days after surgery, during post-intensive care rehabilitation. Autopsy was deferred in this patient and the cause of death is unknown.

Reoperation was necessary in $10(13.9 \%)$ patients. Six of these patients had a wound breakdown or dehiscence which required reoperation; half of these patients had diabetes. Three patients in the reoperation group had caudal adjacent-segment failure. One patient experienced hand grip weakness postoperatively and required a unilateral T1-2 facetectomy and extension of fusion to T3 from T1. Another patient had a T1-2 kyphotic deformity revised by an extension from T4 to T1. Lastly, a patient had a T1-2 fracture which required an extension to T5. All of these patients had reoperations from 7 months to 4.5 years after their initial surgery. The final patient in the reoperation series sustained a left vertebral artery injury after the anterior portion of the procedure. Once the anterior portion of the surgery was completed, the patient was taken to the angiography suite immediately, where occlusion of the left vertebral artery was visualized and the decision was made to coil embolize the vessel. The patient woke up without neurological deficits and was taken to surgery 2 days later for completion of the posterior portion of the surgery.
TABLE 2. Surgical details

\begin{tabular}{lc}
\hline & Value \\
\hline Total no. of patients & 72 \\
\hline Postfusion to C7 & $5(6.9 \%)$ \\
\hline Postfusion to T1 & $42(58.3 \%)$ \\
\hline Postfusion below T1 & $18(25.0 \%)$ \\
\hline Other & $7(9.7 \%)$ \\
\hline Surgical approach & \\
\hline Anterior-posterior & $62(86.1 \%)$ \\
\hline Posterior-anterior-posterior & $10(13.9 \%)$ \\
\hline Massive blood loss (>2 L) & 0 \\
\hline Blood loss, ml & $613 \pm 439$ \\
\hline Operative duration, hrs & $7.83 \pm 1.91$ \\
\hline Vertebral artery injury & $1(1.4 \%)$ \\
\hline Durotomy & 0 \\
\hline RLN injury & $2(2.8 \%)$ \\
\hline
\end{tabular}

Values are presented as number of patients (\%) or mean \pm SD.

Major perioperative complications were defined as a permanent neurological deficit, RLN injury, vascular injury, tracheostomy, PEG tube, stroke, or death. The overall risk of a major perioperative complication was $30.6 \%$. Minor perioperative complications were defined as a temporary neurological deficit, dysphagia, DVT, PE, UTI, pneumonia, or wound infection. The overall risk of a minor perioperative complication was $80.6 \%$.

\section{Discussion}

Smith et al. have reported a series of 78 patients with cervical deformity, 29 of whom required anterior-posterior fixation. ${ }^{21}$ In that series there was a reported $43.6 \%$ complication rate for included patients; $28.2 \%$ of patients had at least one minor complication and $24.4 \%$ had at least one major complication. ${ }^{21}$ In comparison, the minor and major complication rates for our patients were $80.6 \%$ and $30.6 \%$, respectively. Hart et al. reported a retrospective analysis of a 13-patient cohort undergoing anterior and posterior cervical fusion, with $69 \%$ of the patients having at least one complication; however, likely due to the small sample size, these investigators were unable to positively correlate risk factors with complication rates. ${ }^{9}$ Mummaneni et al. have also described their experience in a report of 17 patients undergoing circumferential fusion for cervical kyphotic deformity, with an overall complication rate of $33.3 \%$ (major and minor). ${ }^{13}$

In this study we retrospectively analyzed a single surgeon's experience addressing single-stage, same-day anterior-posterior complex cervical spine deformity correction. Major perioperative complications, as previously defined, occurred with $30.6 \%$ frequency. The incidence of minor complications in our series of patients was $80.6 \%$. In addition to one perioperative death, significant major perioperative complications included prolonged intubation (mean 3.6 days) and eventual tracheostomy (12.5\%). Previous studies have associated airway edema with preoperative smoking status, pulmonary pathology, duration of surgery over 10 hours, and administration of excessive intravenous 
fluids. ${ }^{6,10}$ Due to the prolonged nature of this surgery, which may lead to difficulties with emergent intubation secondary to extensive neck dissection, and according to a protocol based on the senior surgeon's prior experience, patients were kept intubated overnight. Although some of these patients may have been candidates for extubation immediately after surgery, this possibility was not investigated due to the retrospective nature of our analysis. Evaluation for extubation immediately after surgery is something we may consider in the future that may allow us to study the difference in complication rates, particularly with regard to duration of intubation and need for tracheotomy in early compared with delayed extubation groups. The lack of prior studies reporting the incidence of tracheotomy after these complex surgeries precluded us from comparing our results with those of previous studies.

Dysphagia is one of the most common complications of anterior-posterior cervical fusion. Reinard et al. retrospectively evaluated a cohort of 77 patients with an overall incidence of dysphasia of $37.7 \%$ and found that significantly associated risk factors were a left-sided approach and an anterior construct that extends above C4..$^{5}$ Dobhoff tubes were placed in 27 patients in our analysis and kept in place for an average of 13.3 days, allowing the patients maximal opportunity for weaning. Significant dysphagia leading to PEG tube placement affected $12.5 \%$ of our patients (9 patients total; 1 patient had preoperative PEG tube placement). In looking at patients who had a Dobhoff tube in place versus patients who did not (with the exclusion of the 1 patient who had a preoperative PEG), there was a statistically significant association of dysphagia with the duration of intubation, 5.7 versus 2.3 days $(p=0.002)$. All patients who went on to PEG tube placement had a Dobhoff tube in place. This is in comparison to the $15 \%$ rate of PEG tube placement reported by Hart et al., while Chen et al. reported a $38.5 \%$ incidence of PEG tube placement in a cohort of 30 consecutive combined anterior-posterior cervical spine surgeries. ${ }^{3,9}$

The presence of 2 (2.8\%) temporary neurological deficits and the lack of any permanent neurological deficit in this cohort supports the neurological safety of these singlesession circumferential decompression surgeries. In comparison, a review of the literature by Etame et al. found a collective neurological deficit rate of $13.5 \%$.

The significant morbidity outlined in this review begs the question of whether such surgeries should be done in a staged fashion. Although to our knowledge no randomized trials have compared same-day versus staged anteriorposterior cervical spine reconstruction, Siemionow et al. described a retrospective study comparing complication rates between same-day versus staged anterior and posterior cervical decompression and fusion. ${ }^{18}$ In their series, 12 patients underwent same-day surgery and 25 underwent staged surgery, and there were no significant differences between the number of anterior levels fused, total surgical time, or estimated blood loss. ${ }^{18}$ Statistically significant differences were notable for an increase in the number of levels fused posteriorly, total intravenous fluid administration, and length of stay in the staged group..$^{18}$

In summary, there are significant complications associated with complex anterior-posterior multilevel cervical fusions, which raises the question of whether staging this
TABLE 3. Postoperative complications

\begin{tabular}{lc}
\hline & Value \\
\hline Complication & \\
\hline Temporary neurologic deficit & $2(2.8 \%)$ \\
\hline Permanent neurologic deficit & 0 \\
\hline Dysphagia & $45(62.5 \%)$ \\
\hline PEG & $9(12.5 \%)$ \\
\hline Dobhoff tube, days & 13.3 \\
\hline Intubation postop, days & 3.6 \\
\hline Tracheostomy & $9(12.5 \%)$ \\
\hline DVT & $1(1.4 \%)$ \\
\hline PE & 0 \\
\hline Pneumonia & $5(6.9 \%)$ \\
\hline UTI & $1(1.4 \%)$ \\
\hline Superficial wound infection & $4(5.6 \%)$ \\
\hline Stroke & 0 \\
\hline Death & $1(1.4 \%)$ \\
\hline Reoperation & $10(13.9 \%)$ \\
\hline Major postop complications & $22(30.6 \%)$ \\
\hline Minor postop complications & $58(80.6 \%)$ \\
\hline
\end{tabular}

Values are presented as number of patients (\%) unless otherwise indicated.

operation would help lessen the overall risk. However, as discussed previously, there is a lack of high-quality literature supporting one approach versus the other. Thus it remains to be determined if the high complication rate may be partly due to the inherent complexity of the procedure itself and may not vary whether these surgeries are staged or not. Dysphagia in particular remains one of the most prominent complications following multilevel anterior cervical fusion, even in primary surgeries and especially in the older age group. Many of the patients undergoing these procedures, as demonstrated in the case example we present, are undergoing revision surgeries, which entail a higher risk of perioperative dysphagia regardless of other possible contributing factors. There is anecdotal evidence to suggest that fusion extending to $\mathrm{C} 2$ anteriorly and placement of a long anterior plate, especially above the $\mathrm{C} 4$ level, have been correlated with significantly higher risks of dysphagia. There has been recent interest in avoiding extension of the anterior fusion to the $\mathrm{C} 2-3$ disc space unless $a b-$ solutely necessary. Evidence supports utilization of cervical standalone implants, either along the whole construct or above $\mathrm{C} 4$, to decrease the chances of dysphagia. ${ }^{3,17}$ Further, a prolonged anterior approach lasting more than 3 hours, particularly with longus coli muscle retraction, is likely responsible for higher prevertebral soft-tissue swelling and resultant higher rates of dysphagia and prolonged intubation. Use of narrow blades and intermittent longus retraction may help obviate some of the complications. Though not proven to be effective in any high-quality studies, these are some of the possible strategies that we have adopted to lessen the chances of dysphagia and overall complication rates for these complex surgeries.

This study is limited by its retrospective nature. The cohort is that of a single surgeon and while this may help to decrease the variability in treatment paradigms, these same 
treatment strategies may not be generalizable. ${ }^{19}$ While the lack of long-term clinical outcome data is another limitation of the study, the goal of this study was to assess and emphasize the perioperative morbidity following such procedures, as the clinical effectiveness of these surgeries is well recognized in the literature.

\section{Conclusions}

Anterior-posterior cervical decompression and reconstruction is an inherently morbid procedure, but it is necessary in select patients. In this study cohort, which is to our knowledge the largest reported single-surgeon series to date, the overall reoperation rate was $13.9 \%$ and there was a $30.2 \%$ risk of major complications and an $80.6 \%$ risk of a minor complications. The inherent risk of anterior-posterior cervical spine reconstruction is high, but the risks associated with this procedure must be weighed against the unknown risk of anterior-only versus posterior-only strategies for this complex subset of patients. Further studies may elucidate the subtleties of minimizing risk and comorbidities while improving outcomes in this challenging patient population.

\section{References}

1. Bohlman HH, Emery SE, Goodfellow DB, Jones PK: Robinson anterior cervical discectomy and arthrodesis for cervical radiculopathy. Long-term follow-up of one hundred and twenty-two patients. J Bone Joint Surg Am 75:1298-1307, 1993

2. Bolesta MJ, Rechtine GR II, Chrin AM: Three- and four-level anterior cervical discectomy and fusion with plate fixation: a prospective study. Spine (Phila Pa 1976) 25:2040-2046, 2000

3. Chen CJ, Saulle D, Fu KM, Smith JS, Shaffrey CI: Dysphagia following combined anterior-posterior cervical spine surgeries. J Neurosurg Spine 19:279-287, 2013

4. Emery SE, Bohlman HH, Bolesta MJ, Jones PK: Anterior cervical decompression and arthrodesis for the treatment of cervical spondylotic myelopathy. Two to seventeen-year follow-up. J Bone Joint Surg Am 80:941-951, 1998

5. Emery SE, Fisher JR, Bohlman HH: Three-level anterior cervical discectomy and fusion: radiographic and clinical results. Spine (Phila Pa 1976) 22:2622-2625, 1997

6. Emery SE, Smith MD, Bohlman HH: Upper-airway obstruction after multilevel cervical corpectomy for myelopathy. J Bone Joint Surg Am 73:544-551, 1991

7. Etame AB, Wang AC, Than KD, La Marca F, Park P: Outcomes after surgery for cervical spine deformity: review of the literature. Neurosurg Focus 28(3):E14, 2010

8. Gros A, Holzapfel L, Marqué S, Perard L, Demingeon G, Piralla $\mathrm{B}$, et al: Intra-individual variation of the cuff-leak test as a predictor of post-extubation stridor. Respir Care 57:20262031, 2012

9. Hart RA, Tatsumi RL, Hiratzka JR, Yoo JU: Perioperative complications of combined anterior and posterior cervical decompression and fusion crossing the cervico-thoracic junction. Spine (Phila Pa 1976) 33:2887-2891, 2008

10. Kwon B, Yoo JU, Furey CG, Rowbottom J, Emery SE: Risk factors for delayed extubation after single-stage, multi-level anterior cervical decompression and posterior fusion. J Spinal Disord Tech 19:389-393, 2006

11. Lee JS, Youn MS, Shin JK, Goh TS, Kang SS: Relationship between cervical sagittal alignment and quality of life in ankylosing spondylitis. Eur Spine J 24:1199-1203, 2015

12. Miller RL, Cole RP: Association between reduced cuff leak volume and postextubation stridor. Chest 110:1035-1040, 1996
13. Mummaneni PV, Dhall SS, Rodts GE, Haid RW: Circumferential fusion for cervical kyphotic deformity. J Neurosurg Spine 9:515-521, 2008

14. Petrucci N, De Feo C: Lung protective ventilation strategy for the acute respiratory distress syndrome. Cochrane Database Syst Rev 2:CD003844, 2013

15. Reinard KA, Cook DM, Zakaria HM, Basheer AM, Chang VW, Abdulhak MM: A cohort study of the morbidity of combined anterior-posterior cervical spinal fusions: incidence and predictors of postoperative dysphagia. Eur Spine J 25:20682077, 2016

16. Shamji MF, Ames CP, Smith JS, Rhee JM, Chapman JR, Fehlings MG: Myelopathy and spinal deformity: relevance of spinal alignment in planning surgical intervention for degenerative cervical myelopathy. Spine (Phila Pa 1976) 38 (22 Suppl 1):S147-S148, 2013

17. Shao H, Chen J, Ru B, Yan F, Zhang J, Xu S, et al: Zero-profile implant versus conventional cage-plate implant in anterior cervical discectomy and fusion for the treatment of degenerative cervical spondylosis: a meta-analysis. J Orthop Surg Res 10:148, 2015

18. Siemionow K, Tyrakowski M, Patel K, Neckrysh S: Comparison of perioperative complications following staged versus one-day anterior and posterior cervical decompression and fusion crossing the cervico-thoracic junction. Neurol Neurochir Pol 48:403-409, 2014

19. Smith JS, Klineberg E, Shaffrey CI, Lafage V, Schwab FJ, Protopsaltis T, et al: Assessment of surgical treatment strategies for moderate to severe cervical spinal deformity reveals marked variation in approaches, osteotomies, and fusion levels. World Neurosurg 91:228-237, 2016

20. Smith JS, Lafage V, Ryan DJ, Shaffrey CI, Schwab FJ, Patel AA, et al: Association of myelopathy scores with cervical sagittal balance and normalized spinal cord volume: analysis of 56 preoperative cases from the AOSpine North America Myelopathy study. Spine (Phila Pa 1976) 38 (22 Suppl 1):S161-S170, 2013

21. Smith JS, Ramchandran S, Lafage V, Shaffrey CI, Ailon T, Klineberg E, et al: Prospective multicenter assessment of early complication rates associated with adult cervical deformity surgery in 78 patients. Neurosurgery 79:378-388, 2016

22. Vaccaro AR, Falatyn SP, Scuderi GJ, Eismont FJ, McGuire RA, Singh K, et al: Early failure of long segment anterior cervical plate fixation. J Spinal Disord 11:410-415, 1998

23. Wang JC, McDonough PW, Kanim LE, Endow KK, Delamarter RB: Increased fusion rates with cervical plating for threelevel anterior cervical discectomy and fusion. Spine (Phila Pa 1976) 26:643-647, 2001

\section{Disclosures}

Vincent C. Traynelis is a consultant for Medtronic, NuVasive, and Thompson Surgical. He is a patent holder with and receives royalties from Medtronic. Institutional Spine Fellowship support is received from $\mathrm{AO}, \mathrm{NREF}$, and Globus.

\section{Author Contributions}

Conception and design: Wewel, Kasliwal, Traynelis. Acquisition of data: Brahimaj, Wewel, Kasliwal. Analysis and interpretation of data: all authors. Drafting the article: Brahimaj, Wewel. Critically revising the article: Wewel, Kasliwal, Traynelis. Reviewed submitted version of manuscript: all authors. Approved the final version of the manuscript on behalf of all authors: Brahimaj. Statistical analysis: Brahimaj, Wewel. Administrative/technical/material support: Traynelis. Study supervision: Traynelis.

\section{Correspondence}

Bledi C. Brahimaj: Rush University Medical Center, Chicago, IL. bledi_c_brahimaj@rush.edu. 